\title{
CNS stimulation: Effects of stimulus parameters on cardiovascular changes and behavior in the rabbit
}

\author{
W. LLOYD MILLIGAN and D. A. POWELL \\ Neuroscience Laboratory, Veterans Administration Hospital, Columbia, South Carolina 29201
}

\begin{abstract}
In previous experiments of centrally elicited cardiovascular changes in rabbits, relatively high pulse frequencies and short train durations resulted in heart rate (HR) decreases and blood pressue (BP) increases at a variety of diencephalic sites. In the present experiment, stimulus intensity, train duration, and pulse frequency were varied to determine whether other kinds of changes might be obtained with different parameters. The results indicated that, even at low frequencies of stimulation $(10 \mathrm{~Hz})$, the $\mathrm{HR}$ response in the rabbit is a deceleration while BP and respiration rate show concomitant increases. Moreover, as pulse frequency was increased (to about $200 \mathrm{~Hz}$ ), autonomic responses increased in magnitude. HR decelerations and BP increases were obtained at all diencephalic placements regardless of site unless striate activity occurred. Data from behavioral experiments suggested that ESB, which elicited autonomic changes, was either ambiguous or aversive.
\end{abstract}

Electrical stimulation of the diencephalon produces a variety of behavioral and physiological effects. For example, stimulation of the lateral hypothalamus elicits eating and drinking (Valenstein, Cox, \& Kakolewski, 1970) and has rewarding motivational effects (Olds \& Olds, 1963). In addition, this stimulation produces various kinds of autonomic changes (Malmo, 1961, 1964; Perez-Cruet, Black, \& Brady, 1963, Sideroff, Schneiderman, \& Powell, 1971). The role of the cardiovascular system in the mediation of motivational and behavioral changes produced by diencephalic ESB has been the subject of several investigations in the rat (Malmo, 1961, 1964; Perez-Cruet et al., 1963), cat (Abrahams, Hilton, \& Zbrozyna, 1960, 1964), and rabbit (Ban, 1966; Powell, Goldberg, Dauth, Schneiderman, \& Schneiderman, 1972). Although earlier investigators reported obtaining various kinds of sympathetic and parasympathetic responses with diencephalic stimulation in the cat and rat (e.g., Abrahams et al., 1960, 1964; Akert, 1961; Ban, 1966; Fernandez de Molina \& Hunsperger, 1962; Malmo, 1961, 1964), Powell et al. (1972) found mainly BP increases and HR decreases in the rabbit. In the latter experiment, short pulse-train high-frequency stimulation was administered to a chronic unanesthetized preparation. Moreover, although several of the early studies suggested that different cardiovascular response topographies might be associated with different stimulus parameters, these parameters-notably frequency and intensity-were sometimes confounded (e.g., Hare \& Geohegan, 1939).

It thus appears that a systematic investigation of the effects of stimulus variables upon the rabbit's cardiovascular response is a logical first step in

This experiment was supported by VA Institutional Research Funds, Project No. 5737-01. Reprints may be obtained from the authors, Neuroscience Laboratory, Veterans Administration Hospital, Columbia, South Carolina 29201. investigating the relationship between $\mathrm{HR}$ and $\mathrm{BP}$ changes and concomitantly elicited somatic responses. In the present study, we obtained $\mathrm{HR}$ and BP responses at a number of different diencephalic sites as a function of stimulus parameters. These parameters included (a) stimulus intensity, (b) pulse frequency, and (c) pulse train duration. The behavioral significance of this stimulation was also assessed.

\section{METHOD}

\section{Subjects}

Twenty-four New Zealand albino rabbits weighing approximately $3 \mathrm{~kg}$ each were used as subjects. Both male and female subjects were used. From two to four electrodes were implanted in each animal. From this sample, 33 "active" electrodes were obtained. An "active" electrode was defined as one which elicited autonomic changes of a predetermined amplitude, unaccompanied by overt movement.

\footnotetext{
Surgery

The animals were anesthetized with Nembutal (sodium pentobarbital $-15-20 \mathrm{mg} / \mathrm{kg}$ ) and chloryl hydrate $(150-300 \mathrm{mg} / \mathrm{kg})$ administered IV. Standard stereotaxic techniques were used to implant monopolar stimulating electrodes in lateral and medial hypothalamus, and lateral and medial septal area. Bipolar recording electrodes were also implanted in the hippocampus in four animals. The electrodes consisted of Clay-Adams stainless steel insect pins (shaft diameter $.25 \mathrm{~mm}$; tip diameter $.03 \mathrm{~mm}$ ) insulated to within $.5 \mathrm{~mm}$ of the tip with Formvar. The bipolar electrode tips were separated by $.5 \mathrm{~mm}$. A stainless steel jeweler's screw imbedded in the midline skull about $10 \mathrm{~mm}$ anterior to bregma served as the indifferent electrode. The leads from the stimulating and recording electrodes were connected to an Amphenol socket and the socket secured to the skull with dental cement. A 30-ga Teflon catheter was inserted into the medial ear artery under local Xylocaine anesthesia for BP measurement in 10 of the subjects. These animals were cannulated on the morning of the experiment, and autonomic measurements obtained during the afternoon.
} 


\section{Apparatus}

A Biomedical Electronics square wave stimulator with constant current and isolated output was used for electrical stimulation. Current was constantly monitored on an oscilloscope as the potential difference across a 1,000 -ohm series resistor. The stimulus waveform consisted of a biphasic pulse pair with the duration of the pair $1 / 10$ cycle, irrespective of frequency. By letting the pulse-pair duration equal a constant fraction of the cycle length, the average current was maintained constant across frequencies when the latter parameter was manipulated. Both amplitude and duration of the positive and negative going pulses were equal and there was no delay between pulses within a pair. Train duration was $.5 \mathrm{sec}$ in experiments in which intensity and frequency thresholds were determined. The physiological data were recorded on a Grass Model-5 polygraph.

The HR electrodes consisted of stainless steel safety pins chronically inserted into the skin over the left hind limb and right front leg. Insertion of these pins was not accompanied by obvious discomfort, attempts to dislodge the pins, nor infection. Respiration rate was measured by inserting a Grass thermocouple into the nostril of the animal. Arterial BP was measured by connecting a $S$ tatham pressure transducer to the Teflon cannula in the left medial ear artery. During HR and BP determinations, the subjects were restrained in a standard rabbit restrainer (Gormezano, 1966) and placed inside a deactivated refrigerator shell which served to attenuate outside noises, and also served as an electrical shield. The behavioral data were obtained in a shuttlebox programmed to record time spent in $S^{\mathbf{D}}$ or $S \Delta$ and to record jumps to and away from ESB and/or stimulus changes. This apparatus is more fully described in Sideroff et al. (1971). A constant 80-dB SPL white-noise background served as a masking noise during the determination of both the autonomic and behavioral data. BRS solid state programming apparatus was used to program automatically the presentation of stimuli and the recording of responses. Stimulation and recording equipment were located in a control room adjacent to smaller experimental rooms which contained the deactivated refrigerator and shuttlebox.

\section{Procedure}

The experiment took place in three phases. In the first phase, which began approximately 10 days after surgery, HR thresholds were determined at fixed frequency and train-duration values. During these sessions, the animal, restrained inside the sound-attenuated enclosure, was presented with geometrically increasing intensities of stimulation (beginning at 50 microA) until an HR change of $10 \%$ or greater (compared to a prestimulus baseline) was elicited. If no HR changes were obtained, a maximum of $2 \mathrm{~mA}$ peak-to-peak current in septal threshold determinations and $1 \mathrm{~mA}$ in hypothalamic determinations was used. If EMG responses were elicited, the threshold intensity series was terminated. In all cases in which a threshold response was obtained (33 sites), at least one session was devoted to obtaining an intensity function at $60 \mathrm{~Hz}$. During these sessions, four trials at each of three to six intensity levels were administered employing a $60-\mathrm{Hz} \quad .5-\mathrm{sec}$ train-duration stimulus. The order in which the intensities were administered was determined by reference to a table of random numbers. The intertrial interval was $3 \mathrm{~min}$, unless baseline HR had not returned to prestimulus levels, in which case a longer intertrial interval was employed. Instensity functions were obtained on all animals.

In addition to the intensity threshold determinations, at 10 sites in separate animals, a frequency function was obtained at threshold intensity, at an intermediate intensity, and at the highest intensity which did not elicit skeletal activity (as determined from a simultaneous EMG recording). At each intensity and/or frequency value, at least four determinations were made at random times during the session. From three to six frequencies were employed at each intensity level in each animal. The frequencies used were $10,30,60,120,200$, and $500 \mathrm{~Hz}$. The pulse duration was a constant $1 / 10$ cycle at each frequency.
Biphasic pulse-pair durations corresponding to these frequencies were thus $10,3.3,1.67, .83, .05$, and $.02 \mathrm{msec}$, respectively. In eight animals, threshold and subthreshold intensities were employed and train duration manipulated. During these sessions, .5-, 5-, 10-, 20-, and 60-sec trains were used at a frequency of $60 \mathrm{~Hz}$. As in previous sessions, four trials, randomly distributed throughout the session, were administered at each intensity and train-duration value. Threshold determinations comprised a single session. Subsequently, only a single parameter was typically studied at a given site per session. However, occasionally when frequency or duration parameters were manipulated, two sessions were required to complete the experiment. In animals with only a single active site, the first phase was complete after 4 to 7 sessions run over a period of 2 weeks. In animals with multiple sessions, this phase required up to 3 weeks to complete.

During Phase 2, 10 subjects, all of which revealed HR changes clearly related to the stimulus parameters, were cannulated as described above and BP measurements made. The conditions of Phase 1 were then repeated, including the frequency and duration manipulations. These sessions were identical to those of Phase 1 except that BP measurements were obtained.

Finally, during the third phase, 10 animals, selected from those which participated in Phases 1 or 2 , were placed in the shuttlebox and behavioral measurements were determined. The parameters for this phase included those that elicited autonomic changes during Phases 1 and 2 of the study, and in some cases other parameters as well. During these sessions, the animal was placed in the end of the box with which stimulation was associated and allowed to jump either to the other nonstimulated end or remain on the stimulated side. The ESB switched to the other end of the chamber after a 64-sec period had elapsed, regardless of the subjects' behavior, and switched ends every $64 \mathrm{sec}$ thereafter. The presence of ESB was signaled by a $12-\mathrm{V}$ bulb placed in each end of the box. The interstimulus interval for $.5-\mathrm{sec}$ trains of ESB during this phase ranged from 3 to $5 \mathrm{sec}$.

At the conclusion of the study, the animals were perfused with saline and a $10 \%$ formalin solution and electrode placements verified histologically using Oil Red $\mathrm{O}$ and Hematoxylin as described by Cholewick, Butcher, and Ket tlewell (1968).

\section{Response measurement}

All HR measurements were based upon five-beat intervals measured to the nearest millimeter. Chart speed of the polygraph was $50 \mathrm{~mm} / \mathrm{sec}$. On each trial, the HR baseline consisted of the mean duration of two blocks of five beats occurring prior to stimulation onset; the duration of 60 beats following stimulation onset was then compared with the baseline in 12 blocks of five beats each. This was accomplished by computing percent change from baseline scores and heart rate in beats per minute for each five-beat segment. A mean of four trials was computed as the data at each electrode site for each stimulus condition.

Blood pressure changes were measured in $\mathrm{mm} \mathrm{Hg}$ by calibrating the BP channel of the polygraph to permit pen deflections to be converted into $\mathrm{mm} \mathrm{Hg}$. BP topographies, associated with the HR changes, were obtained by comparing the mean systemic arterial BP at the midpoint of each successive preand poststimulation five-beat interval, and in some cases a beat-by-beat analysis was performed. In any case, the magnitude of the BP change after stimulation was computed by comparing the mean BP changes occurring during the HR baseline period with those occurring in the poststimulation period.

Behavioral data in the shuttlebox were obtained in the form of (a) number of crossings, (b) percent time spent in $S^{D}$ (stimulated end of box) and $\mathrm{S}^{\Delta}$ (nonstimulated end), (c) number of escapes (jumps away from ESB), and (d) number of approaches (jumps toward ESB). 
Table 1

Location of Electrode Tips and Range of Biphasic Current Intensity Required for a $10 \%$ Change in HR from a Prestimulus Baseline at a Frequency of $60 \mathrm{~Hz}$ and a $1 / 2-\mathrm{Sec}$ Pulse Train

\begin{tabular}{llc}
\hline Area & N & $\begin{array}{c}\text { Intensity } \\
\text { in } \mathrm{mA}\end{array}$ \\
\hline Lateral Septal Region & 4 & $1.2-1.8$ \\
Medial Septal Region & 4 & $1.0-2.0$ \\
Preoptic Area & 3 & $.4-.6$ \\
Lateral Hypothalamus & 8 & $.2-.8$ \\
Ventromedial Hypothalamus & 5 & $.15-.3$ \\
Dorsomedial Hypothalamus & 5 & $.15-.4$ \\
Posterior Hypothalamus & 2 & $.4-.6$ \\
Thalamus (Nucleus Reticularis) & 1 & .5 \\
Subthalamus & 1 & .2 \\
\hline
\end{tabular}

\section{RESULTS}

\section{Histology}

The general anatomical area and threshold biphasic current intensity required to produce a $10 \%$ change in this area is listed in Table 1. As can be seen, the threshold intensity was somewhat higher in septal regions than in the hypothalamic areas (uncorrelated $\mathrm{t}=4.48, \mathrm{df}=23, \mathrm{p}<.01)$. There was also a tendency for thresholds of more medial placements to be lower than lateral placements, but this difference was not as clear-cut (uncorrelated $\mathrm{t}=2.17, \mathrm{df}=15, \mathrm{p}<.05$ ). Specific electrode tip placements are shown in Figure 1.

\section{Stimulus Intensity}

Figure 2 shows the average intensity which elicited an HR response from five dorso-medial hypothalamic electrodes (in different animals). This response topography was typical of other placements as well, although the peak amplitude of the response was greater for medial hypothalamic than either lateral hypothalamic or septal placements. Uncorrelated t tests were performed upon the peak-amplitude HR elicited by the highest intensity which did not concomitantly elicit movement for (a) septal placements, (b) medial hypothalamic placements, and (c) lateral hypothalamic placements. These $t$ tests revealed that the peak amplitude was significantly greater in medial hypothalamic placements than in either the septum or lateral hypothalamus $(\mathrm{t}=3.8, \mathrm{df}=17, \mathrm{p}<.01$; and $\mathrm{t}=2.13, \mathrm{df}=17, \mathrm{p}<.05$, respectively). However, differences between responses from the septal and lateral hypothalamic sites were not significant. The HR topography shown in Figure 2 is similar to that found by Malmo (1964) in the rat, and Powell et al. (1972) in the rabbit, although in some cases a beat-by-beat analysis revealed an initial acceleration in HR similar to that reported by Malmo (1964) in the medial septal nucleus. However, the present data did not show a systematic relationship between this initial acceleration and site of stimulation. Figure 3 shows a typical trial in which the HR response was biphasic. This figure shows HR in BPM
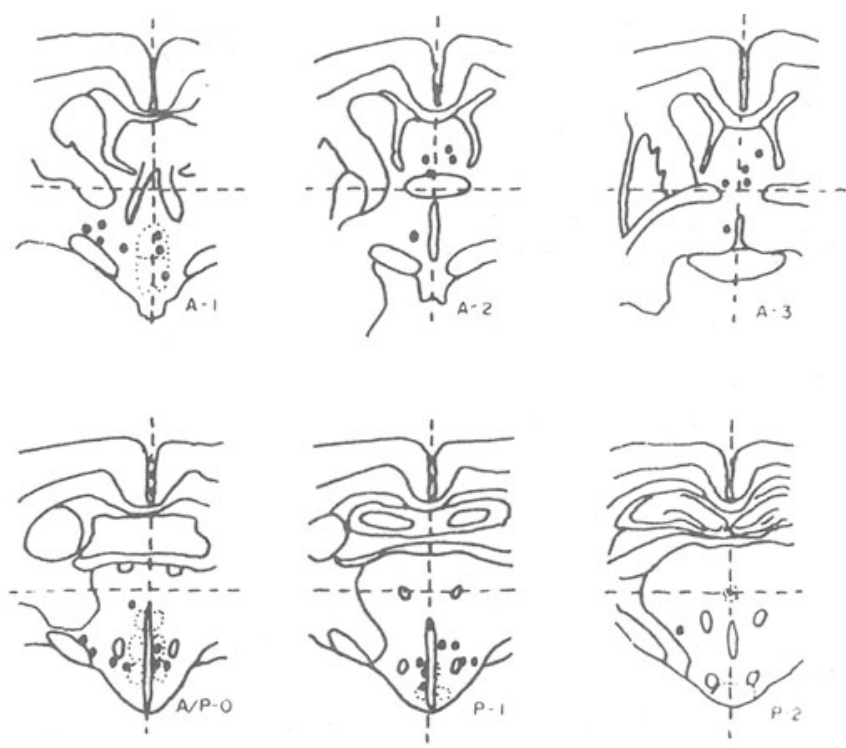

Figure 1. Electrode tip placements (closed circles) superimposed on sections from the Atlas of Sawyer, Everett, and Green (1954).

(left) and BP in $\mathrm{mm} \mathrm{Hg}$ (right) as a function of successive heart beats. The first six poststimulation beats were increases relative to baseline but were followed by the longer duration decelerative response. It is interesting to note that the corresponding blood pressure response is a monophasic increase which begins during the accelerative phase of the HR response.

These cardiovascular changes were also accompanied by increase in respiration rate (RR). RR was measured in five animals, and in all subjects increases in $R R$ were obtained at stimulus intensities that elicited cardiovascular changes. However, baseline RR was highly

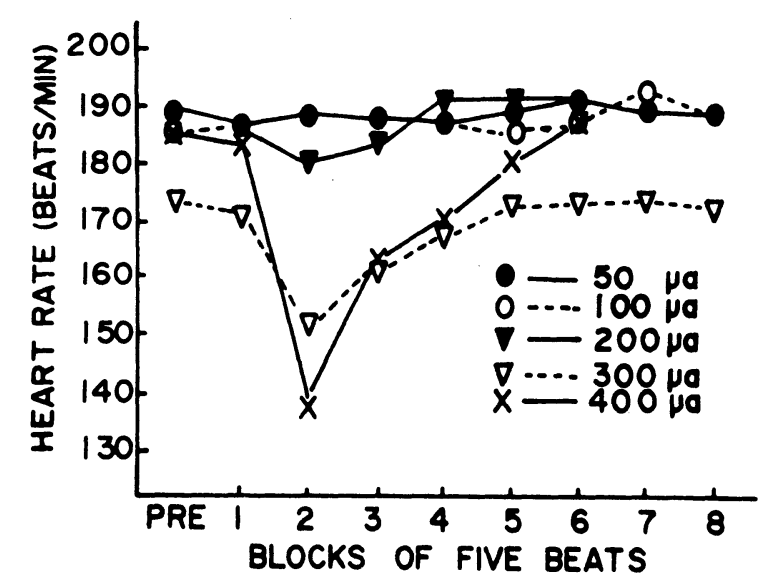

Figure 2. Heart-rate decelerations as a function of poststimulus intervals of five heart beats and intensity of stimulation. Each graph is the average of four trials at each of three dorsomedial hy pothalamic placements in different animals. 


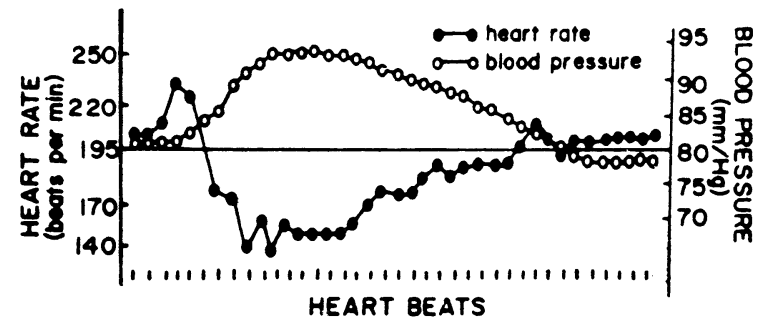

Figure 3. Beat-by-beat heart rate and arterial pressure following $1 / 2-\mathrm{sec}$ ESB trains at 1 microA peak-to-peak to a dorsomedial hypothalamic electrode in a typical animal. The ordinates of the graph have been adjusted to coincide.

variable from trial to trial and much variability in the magnitude of the RR response to stimulation was obtained. Thus, no systematic study of the effects of ESB parameters was performed on the respiration response. In animals in which hippocampal recordings were made, it was found that spiking sometimes occurred at higher levels of stimulation in the septum. The spiking threshold varied from animal to animal, but was, in all cases, considerably higher than that which produced the autonomic changes reported above. It should also be noted that when spiking occurred, it persisted for some time after the cessation of stimulation and thus was not due to stimulus artifact.

\section{Stimulus Frequency}

Stimulus frequency was manipulated as a function of intensity in 10 animals, 6 of which had hypothalamic placements ( 3 medial and 3 lateral) and four of which had septal placements. The effects of frequency were nonmonotonic, as shown in Figure 4, which depicts a typical frequency function obtained from a medial hypothalamic placement. Stimulation at all placements showed that with pulse duration controlled as previously described, the optimum frequency was from 60 to $200 \mathrm{~Hz}$. As shown in Figure 4, smaller magnitude responses were obtained at all placements with both lower and higher frequencies.

\section{Train Duration}

Train duration was studied at two septal and six hypothalamic placements (three medial and three lateral). It was found in several animals that threshold stimulation with a $.5-\mathrm{sec}$ train duration, produced movement responses when the duration was lengthened. Thus only two intensity levels were employed, viz, threshold and subthreshold. The general form of the response was similar regardless of intensity, train duration, or electrode site. Figure 5 shows the HR and BP response during and following a 20-sec ESB train to a lateral hypothalamic site in a typical animal. Stimulus intensity was $300 \mathrm{~mA}$, which was threshold intensity for this animal with a .5 -sec train duration. As can be seen in Figure 5, this response was a pronounced deceleration in HR and increase in BP produced by stimulus onset

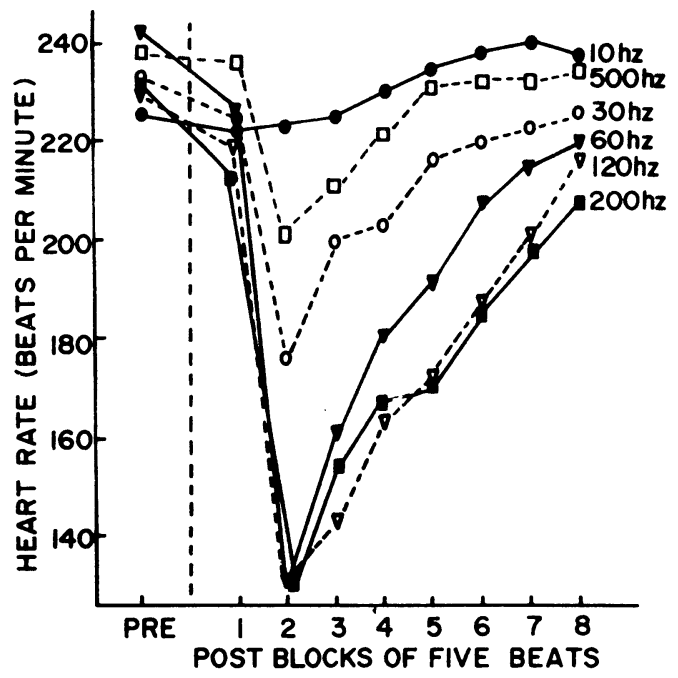

Figure 4. Heart-rate decelerations as a function of poststimulus intervals of five heart beats and frequency of stimulation in a typical hypothalamic placement. Stimulus intensity was 400 microA, peak to peak, and train duration was $.5 \mathrm{sec}$.

followed by further similar changes during stimulation. In addition, stimulus cessation produced a still greater HR deceleration.

\section{Behavioral results}

In the shuttlebox experiments, the animal could jump either toward or away from ESB which was signaled by a light at the appropriate end of the chamber. Results from 10 animals $(2$ septal, 3 medial, and 5 lateral hypothalamic) revealed that at parameters sufficient to elicit the cardiovascular response (i.e., either long trains at low intensities or short trains at high intensities), stimulation was aversive. By aversive, it is meant that there were significantly more jumps away from ESB than toward it. Individual chi squares computed on the escape-vs.-approach frequencies obtained with stimulus parameters at threshold or above for producing cardiovascular changes were all highly significant

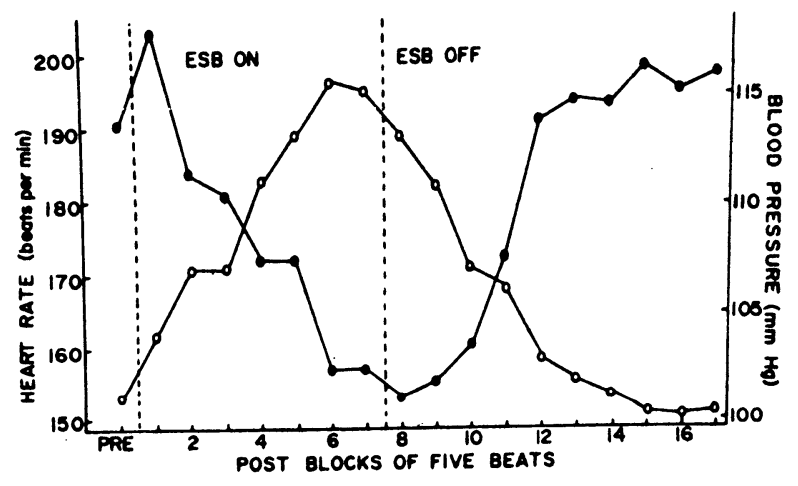

Figure 5. Heart rate (closed circles) and BP (open circles) changes as a function of blocks of five heartbeats following stimulus onset. The stimulus consisted of a $20-\mathbf{s e c}$ train at 300 microA-60 $\mathrm{Hz}$. 
$(p<.01)$. It is possible that stimulation of these sites is rewarding under some other as yet unexplored (perhaps subthreshold) set of stimulating parameters or with other measures of behavior [e.g., barpressing (Powell et al., 1972)]. However, in four animals (two lateral and two medial hypothalamic) stimulus intensity was manipulated in the shuttlebox and behavioral responses obtained. Frequency of jumps away from ESB was positively correlated with the magnitude of the HR response as a function of ESB intensity (see Figure 6a). A similar function of ESB frequency was obtained from four other animals (three lateral and one medial hypothalamic) in the shuttlebox, and again this function was positively correlated with the magnitude of HR response as a function of ESB frequency (see Figure $6 \mathrm{~b}$ ). These findings thus suggest that it is the "aversive" properties of the ESB which are important in mediating the HR response.

\section{DISCUSSION}

The results are consistent with several previous studies of ESB-elicited autonomic changes in the rabbit and rat. The general topography of the HR and BP response is similar to that previously obtained (Powell et al., 1972; Van Dercar, Elster, \& Schneiderman, 1970). The fact that BP increases preceded HR slowing suggests that the latter is a compensatory reaction secondary to the earlier occurring pressor response. Thus, ESB elicited BP increases, via baroreceptors in carotid sinus and aortic arch, act to lower HR by increasing vagal tone to the heart. In prior experiments (Powell et al., 1972) it was found that similar ESB-elicited HR and BP changes with a high-frequency short train-duration stimulus were abolished at appropriate dosages of phentolamine, an alpha adrenergic blocking agent which antagonizes sympathetic vascular tone. These data thus support the hypothesis that ESB-elicited HR decreases are mainly reflex responses to sympathetically induced $\mathrm{BP}$ increases. It is perhaps of note that in none of the 10 animals in the present study, with placements in both medial and lateral septal and hypothalamic areas, were $\mathrm{BP}$ decreases obtained and in all subjects the BP response preceded the decelerative component of the HR response. Of related interest is the finding that neither lower nor higher frequencies of stimulation or longer train durations produced different results, viz, HR increases or BP decreases. Thus, even when stimulus parameters were varied, the basic topography of the HR and BP responses did not change, but was merely increased or diminished in magnitude.

These findings in the unanesthetized rabbit may be contrasted with those of other investigators in anesthetized preparations where different combinations of autonomic changes have been obtained, depending upon site of stimulation (Ban, 1966). It has also been reported that ESB-elicited BP increases can be reversed
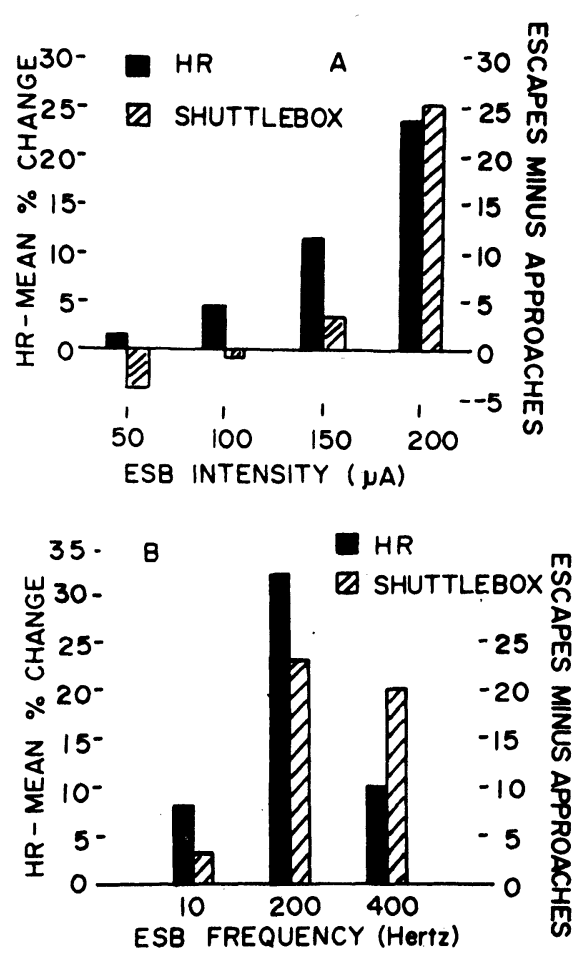

Figure 6. Shut tlebox responses (open bars) and heart-rate decreases (solid bars) as a function of ESB intensity in a medial hypothalamic placement (a, top) and frequency in a lateral hypothalamic placement (b, bottom). This correspondence is also typical of that found in other animals.

to depressor responses if pulse frequency is lowered (Pitts, Larrabee, \& Bronk, 1941). However, these results were not obtained in the present study. Interestingly, the early studies showing BP reversals at low frequencies reported that the depressor responses were maximum at very low frequencies (e.g., $2 \mathrm{~Hz}$ ) and high intensities of stimulation (Pitts et al., 1941; Berry, McKinley, \& Hodes, 1941; Hare \& Geohegan, 1939). Francis, Sampson, Gerace, \& Schneiderman (1973) found, in the unanesthetized rabbit, that all four combinations of BP and HR changes were obtained with diencephalic stimulation at long train durations $(10 \mathrm{sec})$ and a .25 -msec pulse width stimulus. These different response patterns were obtained at several frequencies of stimulation from 25 to 200 pps. Pulse frequency was not manipulated as a function of train duration in the present study, and at none of the eight placements in which train duration was studied were BP decreases or HR increases obtained. Reference to the histological results of Francis et al. (1973) shows that most of their placements were anterior to those studied in the train-duration experiment of the present study (two septal and six hypothalamic). We have also recently obtained data (Powell, Tkacik, \& Buchanan, in preparation) demonstrating both $\mathrm{HR}$ and $\mathrm{BP}$ reversals as a function of very low stimulating frequencies at anterior diencephalic placements. However, we have found that lower frequency $(5 \mathrm{~Hz})$ high-intensity 
stimulation was more effective in eliciting these reversals; in many cases, reversals did not occur at higher frequencies $(50 \mathrm{~Hz})$ even though the train duration was up to $5 \mathrm{sec}$ in duration. Precipitous BP decreases were obtained twice in the present experiment under long durations $(1 \mathrm{~min})$. However, both these responses were associated with pronounced movement and EMG activity, which preceded the BP changes.

As discussed earlier (Powell et al., 1972), the type and degree of anesthesia may also account for discrepancies between previous studies on anesthetized animals and results obtained in unanesthetized subjects. Francis et al. (1973), for example, found that anesthetization attenuated ESB-elicited cardiovascular changes in the rabbit. Moreover, Lico, Hoffman, and Covian (1968) found depressor responses, as opposed to the pressor responses obtained in the present study, by stimulating the septum of the anesthetized rabbit. Abrahams et al. $(1960,1964)$, on the other hand, reported that BP increases and tachycardia accompanied by vasodilation of the limbs was elicited from diencephalic sites in anesthetized cats. These investigators discussed their findings as accompaniments of the classical "defense" reaction which was elicited from similar diencephalic and midbrain sites by Hess (1928) and others (Fernandez de Molina \& Hunsperger, 1962). A possible explanation of the contrasting HR increases reported by Abrahams et al. (1960) in the cat and the HR decreases reported here in the rabbit, may be related to species differences, as discussed elsewhere (Kazis, Milligan, \& Powell, 1973).

The optimum frequency for eliciting $\mathrm{HR}$ and BP responses was $60-200 \mathrm{~Hz}$, which is also optinum for eliciting rewarding operant behavior (Poschel \& Ninteman, 1971; Valenstein, 1964). However, stimulation used in the present experiments was clearly aversive and was most aversive at parameters which were optimum for eliciting the autonomic responses. Although ESB has been used to reinforce barpressing in previous experiments in the rabbit (Bruner, 1967; Sideroff et al., 1971), none of the subjects in the present study showed strong approach behavior to ESB even at parameters previously found to be reinforcing. Some procedural explanation of these differing results is likely since several placements in the present study were clearly located in areas from which rewarding effects have been previously obtained (Bruner, 1967). For example, it is known that duration measures of ESB, such as those used here, do not produce identical results to rate (barpress) measures (Valenstein, 1964). Another possible explanation may be related to the relatively long period of time during which animals received high-intensity ESB under the restrained conditions of the present experiment (up to 4 weeks in animals with multiple placements). Such prior training may predispose the subjects toward ambivalence or escape behavior.

\section{REFERENCES}

Abrahams, V. C., Hilton, S. M., \& Zbrozyna, A. W. Active muscle vasodilatation produced by stimulation of the brain stem: Its significance in the defence reaction. Journal of Physiology, 1960, 154, 491-513.

Abrahams, V. C., Hilton, S. M., \& Zbrozyna, A. W. The role of active muscle vasodilatation in the alerting stage of the defence reaction. Journal of Physiology, 1964. 171, 189-202.

Akert, A. Diencephalon. In: D. E. Sheer (Ed.), Electrical stimulation of the brain. Austin, Texas: University of Texas Press, 1961.

Ban, T. The septo-preoptico-hypothalamic system and its autonomic function. In: T. Tokizane and J. P. Schade (Ed.) Progress in brain research: Correlative neurosciences. Part $A$. Fundamental mechanisms. Amsterdam: Elsevier, 1966. Pp. 615-629.

Berry, C., McKinley, W., \& Hodes, R. Reversals of blood pressure responses caused by changes in frequency of brain stem stimulation. American Journal of Physiology, 1941, 135, 338-346.

Bruner, A. Self-stimulation in the rabbit. Journal of Comparative Neurology, 1967, 131, 615-629.

Cholewick, R. W., Butcher, L., \& Kettlewell, N. M. Oil Red O and Hematoxylin: A rapid histologic technic. Physiology and Behavior, $1968,3,585-586$.

Fernandez de Molina, A. F., \& Hunsperger, R. W. Organization of the subcortical system governing defense and flight reactions in the cat. Journal of Physiology, 1962, 160, 200-213.

Francis, J. S., Sampson, L. D., Gerace, T., \& Schneiderman, N. Cardiovascular responses of rabbits to ESB: Effects of anesthetization, stimulus frequency and pulse-train duration. Physiology and Behavior, 1973, 11, 195-203.

Gormezano, I. Classical conditioning. In: J. B. Sidowski (Ed.), Experimental methods and instrumentation in psychology. New York: McGraw-Hill, 1966. Pp. 385-420.

Hare, K., \& Geohegan, W. A. The influences of frequency of hypothalamic stimulation upon the response. American Journal of Physiology, 1939, 126, 524 (Abstract).

Hess, W. R. Stammgarglien-Riezversuch. Bericht uber die gesamte Physiologie, 1928, 44, 554 .

Kazis, E., Milligan, W. L., \& Powell, D. A. Autonomic-somatic relationships: Blockade of heart rate and eyeblink responses. Journal of Comparative and Physiological Psychology, 1973, 84, 98-110.

Lico, M. C., Hoffmann, A., \& Covian, M. R. Autonomic conditioning in the anesthetized rabbit. Physiology and Behavior, 1968, 3, 673-675.

Malmo, R. B. Slowing of heart rate after septal self-stimulation in rats. Science, $1961,133,1128-1130$.

Malmo, $R$. B. Heart rate reactions and locus of stimulation within the septal area of the rat. Science, 1964, 144, 1029-1030.

Olds, M. E., \& Olds, J. Approach-avoidance analysis of rat diencephalon. Journal of Comparative Neurology, 1963, 120, 259-295.

Perez-Cruet, J., Black, W. C., \& Brady, J. V. Heart rate: Differential effects of hypothalamic and septal stimulation. Science, 1963, 140, 1235-1236.

Pitts, R. F., Larrabee, M. G., \& Bronk, D. W. An analysis of hypothalamic cardiovascular control. American Journal of Physiology, 1941, 134, 359-383.

Poschel, B. P., \&inteman, F. W W. Intracranial reward and the forebrain's" serotonergic mechanism: Studies employing para-chlorophenylalanine and para-chloroamphetamine. Physiology and Behavior, 1971, 7, 39-46.

Powell, D. A., Goldberg, S., Dauth, G. W., Schneiderman, E., \& Schneiderman, N. Adrenergic and cholinergic blockade of cardiovascular responses to subcortical electrical stimulation in unanesthetized rabbits. Physiology and Behavior, 1972, 8, 927-936.

Sawyer, C. H., Everett, J. W., \& Green, J. D. The rabbit diencephalon in stereotaxic coordinates. Journal of Com parative Neurology, 1954, 101, 801-824.

Sideroff, S., Schneiderman, N., \& Powell, D. A. Motivational properties of septal stimulation as the US in classical conditioning of heart rate in rabbits. Journal of Comparative and Physiological Psychology, 1971, 74, 1-10.

Vilenstein, E. S. Problems of measurement and interpretation wich reinforcing brain stimulation. Psychological Review, $1964,71,415-437$.

Valenstein, E. S., Cox, V. C., \& Kakolewski, J. W, Reexamination or tne role or the hypothalamus in motivation. Psychological Review, 1970, 77, 16-31.

VanDercar, D. H., Elster, A. J., \& Schneiderman, N. Heart rate classical conditioning in rabbits to hypothalamic or septal US stimulation. Journal of Comparative and Physiological Psychology, 1970, 72, 145-172. 